\title{
Hochfrequente Dünnschicht Schwinger für zukünftige Ultraschall Phased-Array-Sensoren
}

\author{
Henning Heuer, Thomas Herzog, Susan Walter \\ Fraunhofer Institut für Zerstörungsfreie Prüfverfahren, IZFP-D, Maria Reiche Straße. 2, 01109 Dresden \\ henning.heuer@izfp-d.fraunhofer.de
}

\section{Zusammenfassung}

Für die zukünftige Entwicklung von hochfrequenten Phased-Array ${ }^{1}$ Ultraschallsensoren mit Frequenzen oberhalb von $100 \mathrm{MHz}$ sind die konventionellen PZT-Keramiken nur schwierig zu verarbeiten. Insbesondere die Herstellung von sehr homogenen PZT-Schichten mit Dicken von ca. $20 \mu \mathrm{m}$ lässt sich mit den klassischen Dickschichtdruckverfahren sowie Sol-GelTechnologien nicht in der benötigten Qualität realisieren. Zumindest wenn ein kompletter Sensor auf Basis mikrosystemtechnischer Technologien hergestellt werden soll, ist das bleihaltige PZT nicht einfach in den Prozessfluss zu integrieren. Das Aluminiumnitrid besitzt zwar gegenüber den PZT-Keramiken einen um mindestens eine Zehnerpotenz geringeren piezoelektrischen Koeffizienten, ist aber aufgrund seiner CMOS- Kompatibilität und dem bei höheren Frequenzen geringerem Verlustfaktor ein vielversprechendes piezoelektrisches Material.

Aus diesem Grunde wurden Untersuchungen zur Schichtabscheidung von AIN auf Siliziumsubstraten durchgeführt und mittels mechanischer sowie elektroakustischer Messungen optimale Prozessparameter ermittelt. Für die Bewertung der piezoelektrischen Dünnschichten wurde ein einfaches Testlayout entworfen und spezielle Messplätze zur akustischen Untersuchung im Impuls-Echo-Modus aufgebaut. Für die Herstellung zukünftige AIN-basierter Sensoren wurden Technologien zur Realisierung strukturierter Elektroden getestet.

\section{Motivation}

Durch die Entwicklung von hochfrequenten Phased-Array-Ultraschallsensoren sollen die Anwendungsgebiete und Einsatzmöglichkeiten der Ultraschallmikroskopie ergänzt bzw. erweitert werden. Die Ultraschallmikroskopie ist sensitiv für Delaminationen, Risse, Poren und gibt wichtige Informationen über den Zustand bzw. die Qualität des mikrotechnischen Erzeugnisses. Für die Untersuchung mittels Ultraschallmikroskopie werden heutzutage die mikrotechnischen Komponenten in einem Flüssigkeitsbad platziert und mechanisch abgescannt. Ein bzw. mehrere Einelementschwinger können dabei mit unterschiedlichen Frequenzen und Fokuslagen eingesetzt werden.

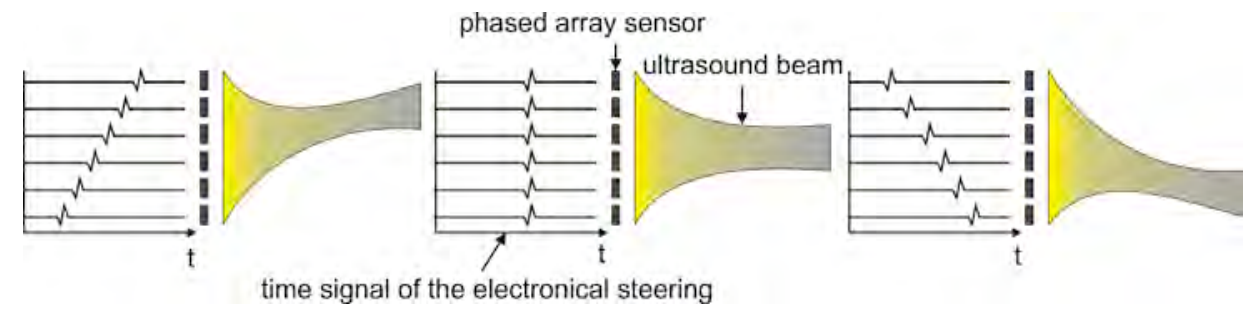

Abbildung 1: Prinzip von Phased-Array Ultraschallsensoren

\footnotetext{
1 Phased Array ... Bei der Phased Array Methode werden die Elemente eines in Linien oder Matrix-Pixel segmentierten Schallwandlers (piezoelektrisches Material) zeitverzögert angesteuert. Durch die Steuerung des Zeitverzuges können Probenvolumen in verschiedenen Winkeln quasi 3-dimensional geprüft werden (siehe Abbildung1).
} 
Es werden Einschwingerprüfköpfe mit Frequenzen von $5 \mathrm{MHz}$ bis zu $400 \mathrm{MHz}$, je nach Prüfobjekt und gewünschter axialer Auflösung verwendet. Problematisch dabei ist, dass die Prüfobjekte in ein Flüssigkeitsbad platziert werden müssen. Zudem ist durch die notwendige mechanische Abrasterung die Messzeit relativ lang und die laterale Auflösung vom scannenden System abhängig.

Durch Verwendung von segmentierten Schallwandlern können mit der Phased-Array-Technik komplexe Probenvolumen 3-dimensional geprüft werden, ohne das der Prüfkopf mechanisch manipuliert werden muss (Abbildung 1). Mit einem einzigen Phased-Array-Prüfkopf kann ein großer Fokusbereich eingestellt werden und unter verschiedenen Winkeln in das Prüfgut eingeschallt werden, wodurch die Messzeit sinkt und die Fehlerauffindwahrscheinlichkeit steigt.

\begin{tabular}{|c|c|}
\hline Ultraschallmikro. & Phased-Array-Technik \\
\hline $\begin{array}{l}\text { Mechanisches Scannen } \\
\text { Hohe Auflösung } \\
\text { Materialcharakterisierung }\end{array}$ & $\begin{array}{l}\text { Elektronisches Schwenken und } \\
\text { Fokussieren } \\
\text { Schnittbilder in Echtzeit } \\
\text { Nur bis } 20 \mathrm{MHz} \text { erhältlich }\end{array}$ \\
\hline \multicolumn{2}{|c|}{ Hochfrequenz Phased-Array-Ultraschall Sensor System } \\
\hline \multicolumn{2}{|c|}{$\begin{array}{l}\text { Reduzierung der Messzeit } \\
\text { Höhere Fehlerauffindwahrscheinlichkeit } \\
\text { Verbesserte Prüfung inhomogener Materialien }\end{array}$} \\
\hline
\end{tabular}

Durch den Einsatz von piezoelektrischen Dünnschichten, die eine hervorragende Kompatibilität zu den Halbleiter- und Mikroelektroniktechnologien aufweisen, ist eine hybrider Sensoraufbau möglich. Die verschiedenen Mikrostruktur- und Trockenätzverfahren können optimal mit den Wafer-Level-Packaging-Technologien verknüpft werden. Auf diese Weise ist die Herstellung hochintegrierter Mehrelementsensoren für Frequenzen oberhalb $100 \mathrm{MHz}$ möglich. Bei Verfügbarkeit derartiger hochfrequenter Ultraschallsensorsysteme könnten die Vorteile beider Techniken verknüpft werden.

\section{Optimierung der Schichtabscheidung und Konzeption einer Teststruktur}

In der Literatur wird die Herstellung von piezoelektrischen Elementen für den Einsatz als mirkomechanisches bzw. mikrooptomechanisches Element auf Basis von AIN beschrieben, auch zum Einsatz als Resonatorbauelement im $\mathrm{GHz}$-Bereich sind mehrere Veröffentlichungen bekannt. Dünnschichten aus mikrokristallinem Aluminiumnitrid (AIN) weisen einen ausgeprägten piezoelektrischen Effekt auf. Voraussetzung ist, das die spezifische

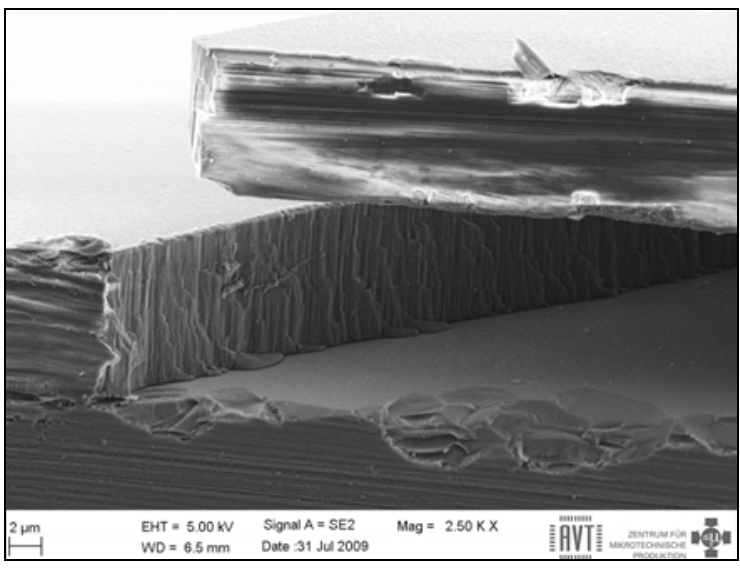

Abbildung 2: AIN-Schicht von $10 \mu m$ Dicke auf Siliziumträger mit deutlicher c-Achsenorientierung
Kristallorientierung dazu eine deutliche c-Achsenorientierung aufweist, sprich die Kristalle möglichst senkrecht auf der Substratoberfläche aufwachsen. Diese Schichten eignen sich besonders zur Herstellung von Sensoren und elektronischen Baugruppen, wie SAW-Filter.

Die bekannten Beschichtungsverfahren haben den Nachteil, dass die maximalen Beschichtungsraten bei ca. $10 \mathrm{~nm} / \mathrm{min}$ für HF-Energieeinspeisung liegen [1, 2]. Für die Herstellung von $10 \mu \mathrm{m}-20$ $\mu \mathrm{m}$ dicken Schichten, wie sie für die hochfrequenten Ultraschallwandler benötigt werden, sind diese Verfahren 
nicht effektiv genug. Durch eine gezielte Variation der Abscheidungsparameter und Analyse der Kristallorientierung mittels XRD sowie durch die elektromechanische Charakterisierung konnten die Schichteigenschaften optimiert werden. Es stellte sich heraus, das für die Abscheidungsparameter nur ein relativ kleines Prozessfenster zu Herstellung von deutlich cAchsenorientierten Schichten mit sehr guten piezoelektrischen Eigenschaften vorhanden ist. Gleichzeitig ist darauf zu achten, dass die inneren Spannungen der abgeschiedenen Schichten nicht zu groß werden, um eine gute Haftung auf dem Elektrodenmaterial bzw. dem Substrat zu erreichen. Für eine Bewertung der Nutzbarkeit von AIN-Schichten wurde zunächst ein einfaches Testlayout als Einelementschwinger entwickelt. Damit war eine Untersuchung der folgenden Punkte möglich:

- Charakterisierung des elektrischen und elektromechanischen Verhaltens der Sensoren

- Optimale Abscheidungsparameter

- Maximal mögliche Schichtdicke

- Haftfestigkeit

- Größe und Beeinflussbarkeit der Schichtspannungen

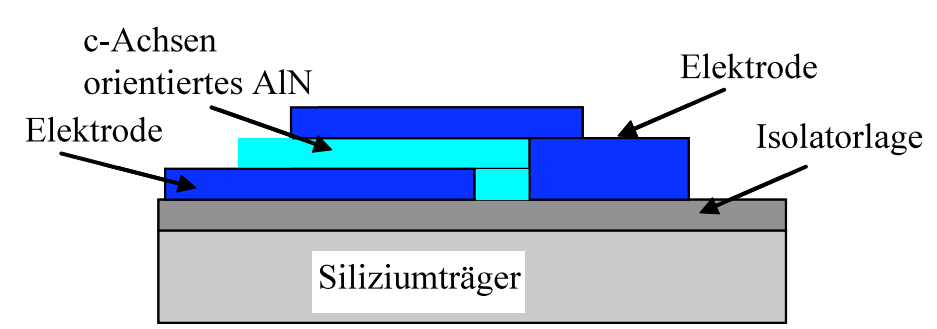

Abbildung 3: Layout der Teststruktur eines einzelnen Schwingers und Querschnitt Die Fläche des abgeschiedene Durchmesser größer dimensioniert worden als die Elektroden selbst, da bei der Beschichtung Randeffekte auftreten, die die folgenden Messungen beeinträchtigen würden. Es wird eine hohe Steifigkeit der Masken benötigt, da die auch auf der Maskenoberfläche abgeschiedenen Schichten hohe mechanische Spannungen erzeugen, die zu einer Verwölbung führen kann. Als Material wurde daher $630 \mu \mathrm{m}$ dickes Aluminiumoxid gewählt.

\section{$4 \quad$ Ergebnisse der Charakterisierung}

Mittels mikroanalytischer Verfahren und anhand der XRD-Messungen sind für die verschiedenen Abscheidungsparameter die jeweiligen Einzelschwinger charakterisiert worden. Wie in Abbildung 4 ersichtlich ist, kann man an bei der XRD-Messung an den gemessenen Peaks erkennen, in welcher Orientierung das AIN auf dem Silizium abgeschieden wurde.
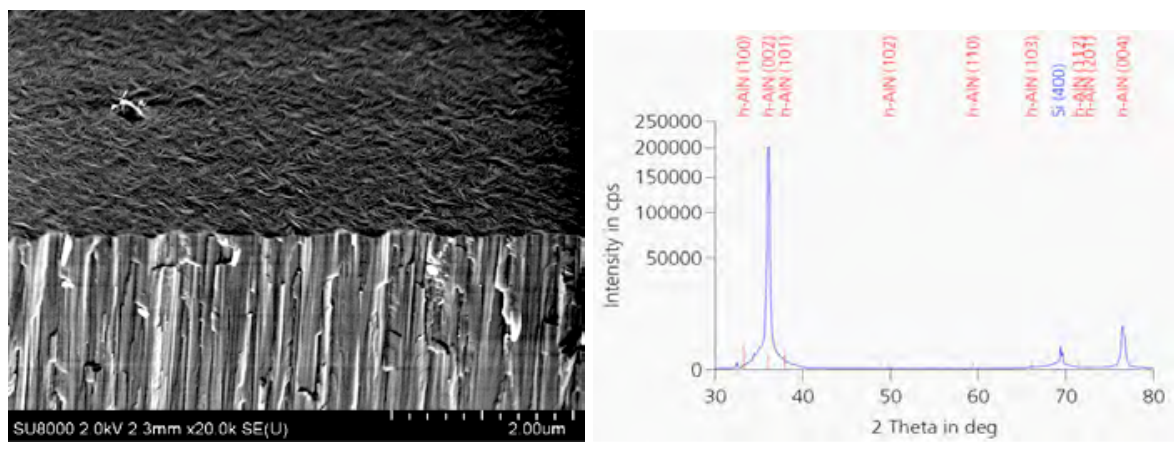

Abbildung 4: Rasterelektronenbild (links) und XRD-Messung (rechts) einer AIN Schicht mit sehr guten piezoelektrischen Eigenschaften, die sich durch eine dichte feinkristalline Struktur mit hoher c-

Achsenorientierung auszeichnet (Quelle: FhG FEP) 
Es konnte ein Zusammenhang zwischen der Kristallstruktur, den XRD-Messungen und den piezoelektrischen Eigenschaften der AIN-Schichten nachgewiesen werden. Eine säulenartige Struktur, die senkrecht zur Substratoberfläche ausgerichtet ist und eine ebene Oberflächenbeschaffenheit weisen auf eine hohe c-Achsenorientierung sowie gute piezoelektrische Eigenschaften hin. Zum Vergleich dazu zeigt Abbildung 5 eine poröse Schicht mit eher geringer c-Achsenorientierung.
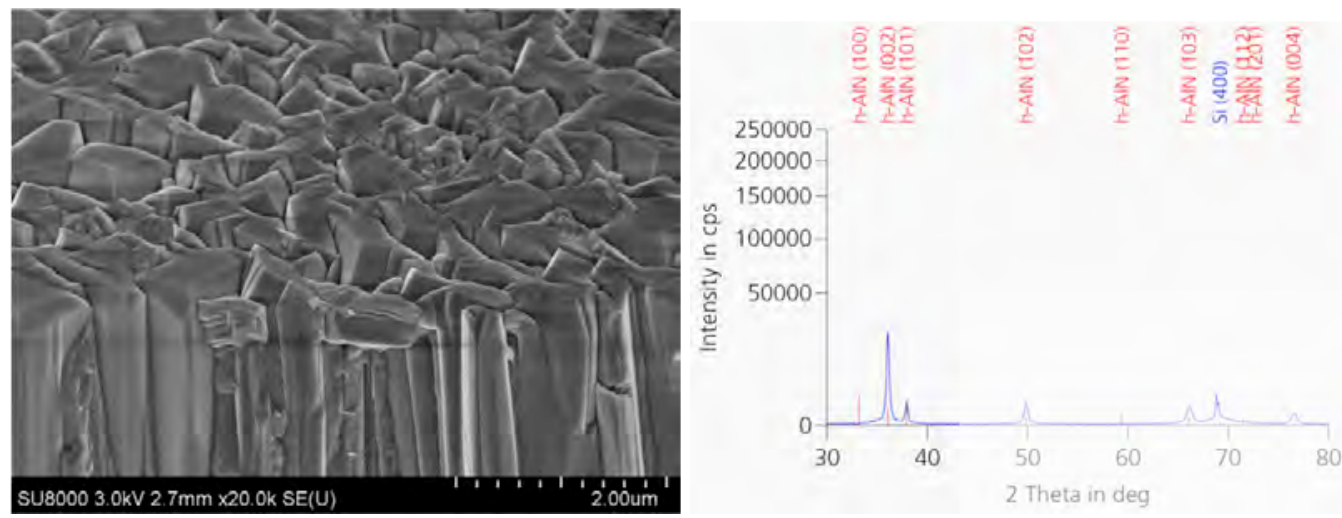

Abbildung 5: Rasterelektronenbild (links) und XRD-Messung (rechts) einer AIN Schicht mit vergleichweise schlechten piezoelektrischen Eigenschaften, die sich durch eine poröse Struktur mit geringerer c-Achsenorientierung auszeichnet (Quelle: FhG FEP)

\section{Elektromechanische Charakterisierung im Impuls-Echo-Modus}

Für piezoelektrische Keramiken wird die elektromechanische Charakterisierung anhand der elektrischen Impedanz-Messung durchgeführt. Aus dem Kurvenverlauf von Betrag und Phase kann direkt die Resonanzfrequenz abgelesen werden. Durch die hier gewählte Materialkombination erhält man für AIN-Schichten nur einen Impedanzverlauf, wie er für dielektrische Materialien typisch ist, die Resonanzfrequenz kann daran nicht abgelesen werden. Für eine Aussage zur Qualität des piezoelektrischen Effektes wurden Messplätze zur akustischen Untersuchung im Impuls-Echo-Modus aufgebaut. Beim Impuls-Echo-Verfahren dient der AINEinzelschwinger als Sender und Empfänger. Die bei Beaufschlagung mit einem elektrischen Spannungsimpuls ausgesendete Schallwelle breitet sich nahezu ungehindert im SiliziumSubstrat aus und trifft dann an der Unterseite auf die Grenzfläche Silizium-Luft, an der die Schallwelle reflektiert wird und wieder zur AIN-Schicht gelangt (siehe auch Abbildung 3). Dort wird dieser mechanische Impuls wieder in ein elektrisches Signal umgewandelt. Im Ergebnis wurden die aus dem Siliziumsubstrat reflektierten Echos, die wiederum ein elektrisches Signal im Schwinger erzeugen, detektiert.

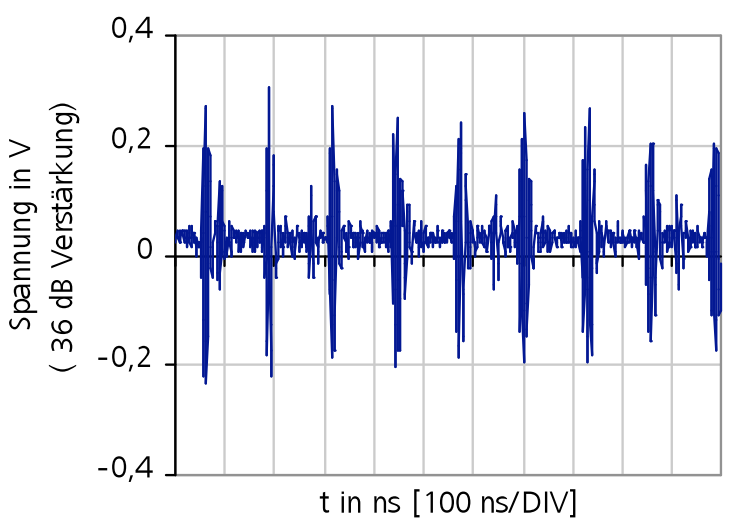

Abbildung 6: Echo-Impulse von der Silizium-Rückwand
Es wurden die AIN-Einelementschwinger an einen Pulsgenerator angeschlossen und mit einem Nadelimpuls angeregt. Der Receiver wurde auf eine Empfangsverstärkung von $36 \mathrm{~dB}$ sowie einem Hochpassfilter zwischen $30 \mathrm{MHz}$ und $500 \mathrm{MHz}$ eingestellt. Der Pulsgenerator liefert ein Signal mit hoher Amplitude (-230V) sowie kurzer Impulsweite (1,5 ns). Man erkennt im Impuls-Echo-Modus deutlich die Rückwandechos (Abbildung 6) von der Siliziumunterseite, die in ihrem zeitlichen

Abstand der Dicke des Siliziums bezogen auf die Schallgeschwindigkeit entsprechen. Auch 
die sehr geringe akustische Dämpfung von Silizium ist aus dem langsamen Abfall der Impulshöhen ersichtlich. Die Einzelschwinger liefern von der oberen Seite, die an Luft grenzt, auf Grund der starken Dämpfung kein Echo in vergleichbarer Höhe. Da die Schalleigenschaften und Dicken der einzelnen Silizium-Wafer annähernd konstant sind, kann man diese Echosignale auswerten, um die gesendete Schallintensität der verschiedenen Schwingerelemente zu vergleichen. Die unterschiedlichen Sende- und Empfangseigenschaften der Schwinger, resultieren aus unterschiedlichen Eigenschaften der AIN-Schichten, folglich aus den verschiedenen Abscheidungsparametern mit ansonsten konstanten geometrischen Abmaßen. Die Schichten konnten nun bezüglich ihrer piezoelektrischen Eigenschaft über die Höhe der jeweiligen Impulsamplituden der Echos

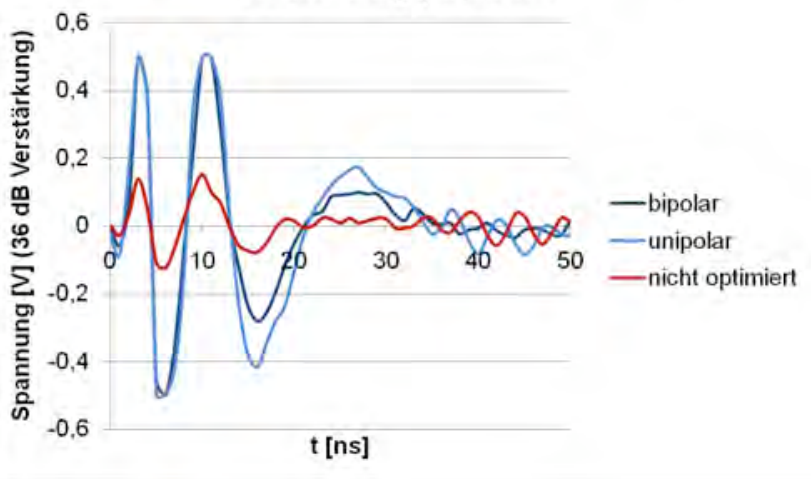

Abbildung 7: Vergleich der gemessenen Signalverläufe des Rückwandechos vom Silizium für Schwinger mit optimierten und nicht optimierten Beschichtungsparametern

ausgewertet und verglichen werden.

Die Variation der Beschichtungsparameter wurden am Fraunhofer FEP durchgeführt und die entstandenen Sensoren am Fraunhofer IZFP charakterisiert. Es konnte gezeigt werden, dass eine Parameteroptimierung zu einer erheblichen Verbesserung der Effektivität der Sensoren beiträgt Abbildung 7. Es wurden zwei Parametersätze für die Beschichtung ermittelt, die zu AINSchichten mit sehr guten piezoelektrischen Eigenschaften

führen (Tabelle 1). Der größte Unterschied der beiden Parametersätze ist der Betriebsmodus der Magnetron-Sputteranlage. Es kann sowohl der unipolare als auch der bipolare Modus verwendet werden, je nach gewünschter oder möglicher Intensität der Bombardierung des Substrates mit den AIN-lonen. Beim unipolaren Modus wird eine gepulste Gleichspannung jeweils zwischen zwei Targets und einer separaten abgeschirmten Anode geschalten. Beim bipolaren Modus werden zwei Targets verwendet und die Spannung mit wechselnder Polarität nur zwischen diesen geschaltet.

Tabelle 1: Optimierte Beschichtungsparameter für c-Achsen orientiertes AIN (Quelle: FEP)

\begin{tabular}{|l|l|l|}
\hline & Unipolar Modus & Bipolar Modus \\
\hline Abscheiderate & $3,5 \mathrm{~nm} / \mathrm{sec}$ & $2,5 \mathrm{~nm} / \mathrm{sec}$ \\
\hline Mech. Spannungen & $-1 \mathrm{GPa}$ & $-2 \mathrm{GPa}$ \\
\hline Durchbruchfeldstärke & $2.3 \mathrm{MV} / \mathrm{cm}$ & $3.1 \mathrm{MV} / \mathrm{cm}$ \\
\hline Kristallorientierung & $002(99,9 \%)$ & $002(99,9 \%)$ \\
\hline Spezf. elektr. Widerstand & $5.3 \mathrm{E}+12 \Omega \mathrm{cm}$ & $1.2 \mathrm{E}+13 \Omega \mathrm{cm}$ \\
\hline Dichte & $3,16 \mathrm{~g} / \mathrm{cm} 3$ & $3,20 \mathrm{~g} / \mathrm{cm} 3$ \\
\hline d33 & $6,5 \mathrm{pm} / \mathrm{V}$ & $7,2 \mathrm{pm} / \mathrm{V}$ \\
\hline Schichtspannung & $-1 \mathrm{GPa}$ & $-2 \mathrm{GPa}$ \\
\hline
\end{tabular}

\section{Strukturierungstechnik für die Elektrodensegmentierung}

Die Herstellung von Phased-Array Ultraschallsensoren auf Basis von piezoelektrischen Dünnschichten erfordert auch die Entwicklung einer Technologie zur Strukturierung der Elektroden. Schwerpunkte der Untersuchungen bildeten die Abscheidung strukturierter Deckelektroden durch maskierte Beschichtung, die Strukturierung mittels Präzisionstrennschleifen (Wafer-Sägen) und die Strukturierung mittels Laserschneiden. Als Wafersäge kam die am IZFP verfügbare Maschine von Typ DISCO DAD 321 zum Einsatz. Es wurden unterschiedliche Trennungsbreiten von $10 \mu \mathrm{m}$ bis $200 \mu \mathrm{m}$ sowie Tiefenvariationen von $5 \mu \mathrm{m}, 7,5 \mu \mathrm{m}$ und $10 \mu \mathrm{m}$ durchgeführt. Eine Strukturierung von $150 \mathrm{~nm}$ Aluminium, ohne das AIN ebenfalls einzusägen ist praktisch nicht möglich. Außerdem ist die Variation des 
Elektrodenlayouts (keine Kreise möglich) und die Abstände durch die Säge sehr eingeschränkt.

Ziel der Experimente mittels Laserschneiden war die Strukturierung der AluminiumDeckelektroden sowie AIN-Schicht in einer Schnitt-Tiefe von $3 \mu \mathrm{m}-5 \mu \mathrm{m}$. Diese Arbeiten wurden an der TU Dresden in Kooperation mit dem IZFP durchgeführt. Es wurde ein Faserlaser mit einer max. Leistung von $20 \mathrm{~W}$ bei einer Schnittgeschwindigkeit von $50 \mathrm{~mm} / \mathrm{s}$ eingesetzt. Im Nachfolgenden sind nur kurz einige Ergebnisse anhand von REM-Aufnahmen dargestellt.

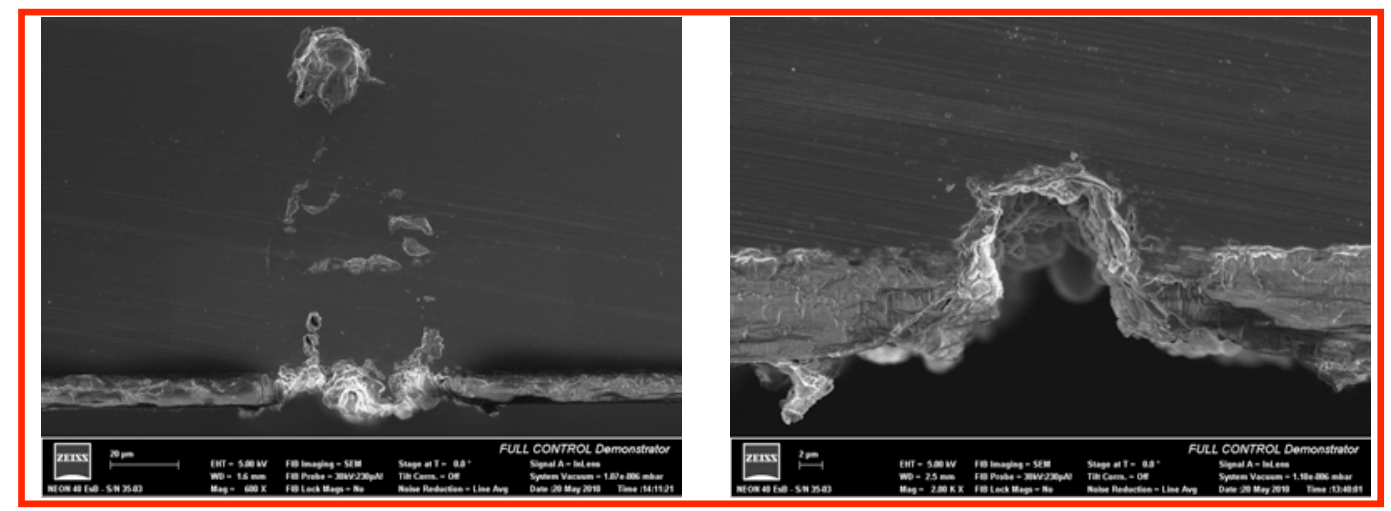

Abbildung 8: Schnittqualität bei Laserparameter 1 kHz, 14 W unzureichend (Quelle: IAVT)

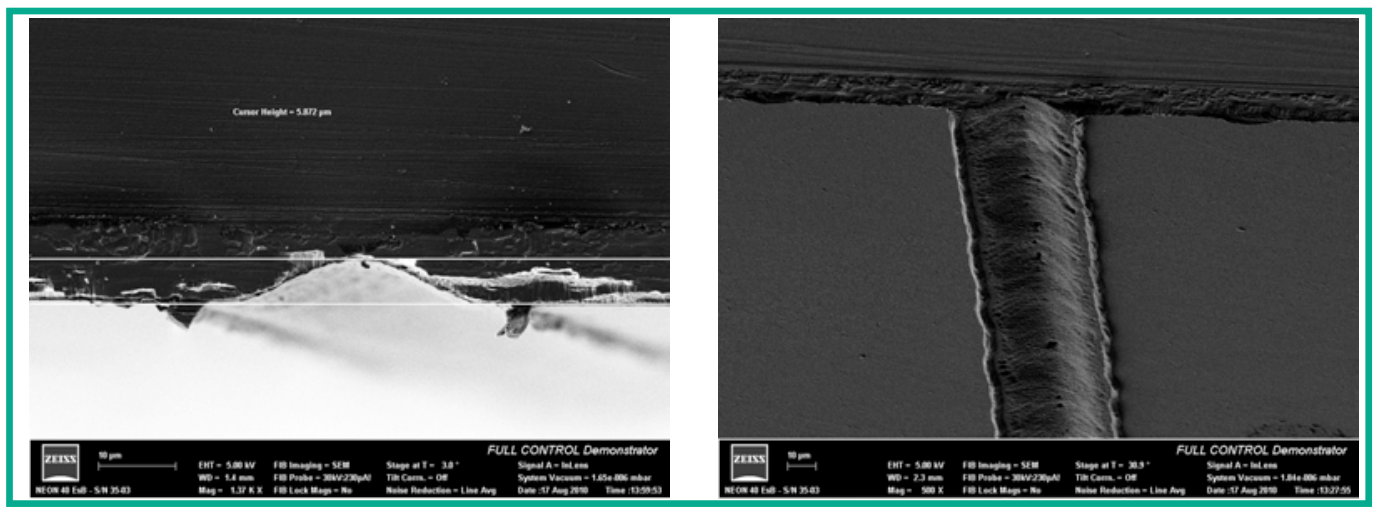

Abbildung 9: Schnittqualität bei Laserparameter 1 kHz, 2 W gut (Quelle: IAVT)

\section{$5 \quad$ Zusammenfassung und Ausblick}

Es wurden grundlegende Untersuchungen an der piezoelektrischen Dünnschicht Aluminiumnitrid (AIN) durchgeführt. Für die Bewertung der piezoelektrischen Dünnschichten wurde ein einfaches Testlayout entworfen und spezielle Messplätze zur akustischen Untersuchung im Impuls-Echo-Modus aufgebaut. Mittels mikroanalytischer Verfahren und anhand der XRD-Messungen sind für die verschiedenen Abscheidungsparameter die jeweiligen Einzelschwinger charakterisiert worden. Es konnte ein Zusammenhang zwischen der Kristallstruktur, den XRD-Messungen und den piezoelektrischen Eigenschaften der AINSchichten nachgewiesen werden. Beim Impuls-Echo-Verfahren dient der AIN-Einzelschwinger als Sender und Empfänger. Es wurden die AIN-Einelementschwinger an einen Pulsgenerator angeschlossen und mit einem Nadelimpuls angeregt. Die unterschiedlichen Sende- und Empfangseigenschaften der Schwinger, resultieren aus unterschiedlichen Eigenschaften der AIN-Schichten, folglich aus den verschiedenen Abscheidungsparametern mit ansonsten konstanten geometrischen Abmaßen. Die Schichten konnten bezüglich ihrer piezoelektrischen Eigenschaft über die Höhe der jeweiligen Impulsamplituden der Echos ausgewertet und verglichen werden. Es wurden zwei Parametersätze für die Beschichtung ermittelt, die zu AINSchichten mit sehr guten piezoelektrischen Eigenschaften führen. Für die Herstellung zukünftige AIN-basierter Sensoren wurden Technologien zur Realisierung strukturierter Elektroden getestet. In weiterführenden Untersuchungen sollen die Einflüsse 
unterschiedlicher Elektrodengrößen, verschiedener Substratmaterialien und Schichtdicken der Schwinger ermittelt werden. Somit sollen verschiedene Varianten eines Designs und unterschiedliche Anwendungsfelder der zukünftigen Phased-Array-Sensoren getestet werden.

\section{Literatur}

[1] J.X. Zhang, Y.Z. Chen, H. Cheng, A. Uddin, Shu Yuana, K. Pita, T.G. Andersson, Thin Solid Films 471 (2005) 336-341.

[2] F. Engelmark, G.F. Iriarte, I.V. Katardjiev, M. Ottosson, P. Muralt, S. Berg, J. Vac. Sci. Technol. A 19 (2001) 2664-2669. 